\title{
Validação de um sistema de previsão para a pinta preta na produç̧ão integrada do tomateiro
}

\author{
Walter Ferreira Becker ${ }^{1}$
}

\begin{abstract}
Resumo - A pinta preta causada por Alternaria spp. é uma das principais doenças do tomateiro ocorrendo em cultivos dessa cultura na microrregião de Joaçaba, a qual é responsável por mais de $57 \%$ da produção do estado de Santa Catarina. Para o controle desta doença, são aplicados fungicidas semanalmente, o que implica em aumento de custos de produção. $\mathrm{O}$ objetivo deste trabalho foi comparar a aplicação preventiva semanal de fungicida com o sistema de previsão TomCast. Esse sistema utiliza a duração do molhamento foliar e a temperatura média neste período para calcular a severidade diária da doença (VSD). As pulverizações baseadas no calendário semanal iniciaram na semana do transplante enquanto as com o previsor TomCast iniciaram quando o valor de VSD acumulado atingiu o limiar estabelecido (10, 15, 20, 25 e 30 VSD). Em cada um dos anos estudados, a pulverização de acordo com o TomCast resultou no controle de doença e na produção comparável com o calendário semanal, mas retardou o início da pulverização em 26 a 46 dias e reduziu o número de pulverizações em até $84 \%$.
\end{abstract}

Termos para indexação: Solanum lycopersicum; sistema de alerta; Alternaria solani.

\section{Validation of a predictive system for the early blight in the integrated production of tomato}

Abstract - Tomato early blight, caused by Alternaria spp., is one of the main diseases occurring on tomato crops in the Joaçaba microregion, which is accountable for more than $57 \%$ of the tomato production in the State of Santa Catarina. Fungicides are commonly used on a weekly schedule to control early blight, increasing production costs. This study aimed to compare the preventive calendar-based application schedules with the TomCast forecast system. This system uses the duration of leaf wetness and average temperature during the wetness period to calculate a daily severity value (DSV). The calendar-based fungicide application started in the week of transplanting, whereas the TomCast predictor fungicide spray begun when the cumulative DSV reaches a predetermined threshold $(10,15,20,25$, and $30 \mathrm{DSV})$. In each of the years studied, timing sprays according to the TomCast predictor resulted in disease control and yield comparable with the weekly schedule, but delayed the initial fungicide spray for as much as 26 to 41 days. Additionally, the TomCast predictor system allowed reducing the number of sprays by up to $84 \%$.

Index terms: Solanum lycopersicum; forecast system; Alternaria solani.

\section{Introduç̧ão}

O estado de Santa Catarina ocupa a sexta posição em área plantada $(2,76$ mil ha), a sétima posição em produção

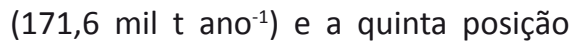
em produtividade $\left(62 \mathrm{t} \mathrm{ha}{ }^{-1}\right)$ do tomate, com uma participação de $4,69 \%$ da produção nacional do consumo in natura. A microrregião de Joaçaba se destaca, com $49,3 \%$ da área plantada e $57,09 \%$ da produção do estado, constituindo a segunda maior região nacional fornecedora de tomate no verão e o principal polo produtor do estado, com destaque ao município de Caçador, com plantio de 700 ha (IBGE, 2017).
Dentre as principais doenças do tomateiro, na microrregião de Joaçaba, destaca-se a pinta preta (FAORO \& TOMASELLI, 1997), causada por Alternaria solani Ell. \& Mart., L.R. Jones \& Grout e espécies relacionadas (RODRIGUES, 2009). A rápida expansão da área cultivada nos últimos anos, aliada ao clima predominantemente chuvoso no verão, pode ter contribuído para o agravamento na intensidade da doença. A doença ocorre nas folhas mais velhas do tomateiro na forma de manchas marrom-escuras; também ocorre no caule e frutos, causando severos danos, e em todos os estádios da planta (BASU, 1974; JONES, 1991) reduzindo consideravelmente a área fotossintetizante e a produtividade.

$\mathrm{O}$ alto potencial destrutivo da doença, aliado a indisponibilidade de genótipos resistentes entre os cultivares tradicionalmente plantados (TÖFOLI et al., 2003; LOPES \& ÁVILA, 2005), faz do controle químico semanal a principal opção entre os produtores de Caçador (SC). Os sistemas de previsão para o tomateiro rasteiro e tutorado são de largo uso em outros países (CAMPBELL \& MADDEN,1990; SIKORA et al., 2002), porém incipientes no Brasil. Em tomateiro, Santos (2000) avaliou o sistema de previsão PAST e obteve uma redução de $30 \%$ nas aplicações de fungicidas, 
enquanto Paul et al. (2004) obtiveram reduções de $60 \%$ das pulverizações. O modelo TomCast (Tomato Forecasting) foi estendido a outras interações patógeno-hospedeiro proporcionando significativa redução de pulverizações (DORMAN et al., 2009).

O objetivo deste trabalho foi avaliar o modelo TomCast na previsão da pinta preta do tomateiro tutorado conduzido em sistema de produção integrada de modo a orientar o produtor do momento de aplicação do fungicida para controle da doença.

\section{Material e métodos}

Os ensaios foram realizados na Epagri - Estação Experimental de Caçador, Caçador-SC, na microrregião de Joaçaba - durante os anos agrícolas 2009/10, 2010/11 e 2011/12. O clima é do tipo $\mathrm{Cfb}$, temperado, com chuvas frequentes no verão (PANDOLFO et al., 2002). Registraram-se as variáveis climáticas (temperatura, molhamento foliar) pelo aparelho termo-higro-umectógrafo, marca Luftt, modelo 8341. Diariamente, o período do molhamento foliar e a temperatura daquele período foram convertidos em valores de severidade da doença (VSD), variando de 0 a 4, que significam uma condição ambiente desfavorável até altamente favorável à doença, respectivamente (Tabela 1; MADDEN et al., 1978). Quando alcançado o somatório pré-determinado para cada VSD, iniciou-se a pulverização do respectivo tratamento.

O delineamento experimental foi inteiramente casualizado, dada a uniformidade da área (topografia, fertilidade e tipo de solo). Utilizou-se o cultivar $\mathrm{Pa}$ ronset com a condução, tratos culturais, controle de pragas e doenças realizadas conforme as indicações técnicas da cultura (MUELLER et al., 2008). As variáveis avaliadas foram submetidas à análise de variância ou, quando não atendidos os pressupostos desta, pelo teste não-paramétrico de Kruskal-Wallis, utilizando o programa estatístico SAEG.

Ensaio 2009. Os tratamentos com quatro repetições foram: 1) pulverização semanal; 2) pulverização no acumulado de 10-VSD (valores diários de severidade da doença); 3) pulverização no

Tabela 1. Valores de severidade diários da doença (VSD) estimados em função da temperatura média ambiente durante o período (horas) de molhamento foliar Table 1. Daily disease severity values (SDV) estimated as a function of the mean ambient temperature during the period (hours) of leaf wetness

\begin{tabular}{llllll}
\hline $\begin{array}{l}\text { Temperatura } \\
\text { média }{ }^{\circ} \mathrm{C}\end{array}$ & 0 & 1 & 2 & 3 & 4 \\
\hline $13-17$ & $0-6$ & $7-15$ & $16-20$ & $21+$ & \\
$18-20$ & $0-3$ & $4-8$ & $9-15$ & $16-22$ & $23+$ \\
$21-25$ & $0-2$ & $3-5$ & $6-12$ & $13-20$ & $21+$ \\
$26-29$ & $0-3$ & $4-8$ & $9-15$ & $16-22$ & $23+$ \\
\hline Fonte: Madden et al. (1978) & & & &
\end{tabular}

acumulado de 15-VSD; 4) pulverização no acumulado de 20-VSD; 5) pulverização no acumulado de 25-VSD; A parcela experimental foi constituída por uma fileira de 16 plantas, sendo 12 úteis, espaçadas de $1,5 \mathrm{~m}$ entre fileiras e $0,5 \mathrm{~m}$ entre plantas, com tutor vertical de fitilho, para uma planta com duas hastes. As parcelas ficaram sujeitas a infecção natural oriunda de tomateiros próximos a área experimental. As mudas de tomate foram oriundas de viveiristas e plantadas 01/12/2009.

A pulverização semanal teve inicio após o plantio enquanto os demais tratamentos iniciaram de acordo com o modelo TomCast (PITBLADO, 1992). Os valores de severidade da doença foram obtidos da relação entre o período de molhamento foliar e a temperatura neste período e o VSD acumulado durante o período de cultivo ( $\Sigma$ VSD) (Tabela 1 ). Para a primeira pulverização, o VSD foi acumulado diariamente até atingir o valor limite de 35; pulverizações subsequentes ocorreram quando o valor VSD, estimado para cada tratamento, era alcançado. A cada pulverização o valor foi zerado, iniciando novo acúmulo de valores e assim sucessivamente durante o ciclo.

Não se utilizou uma testemunha sem fungicida, com o propósito de minimizar o erro experimental, devido à interferência entre as parcelas dos tratamentos (ZADOKS \& SCHEIN, 1979; PAUL et al., 2004). O fungicida boscalida (50\% i.a.) foi aplicado na dosagem de $7,5 \mathrm{~g}$ i.a. $\mathrm{hl}^{-1} \mathrm{em}$ alto volume (1000 $\mathrm{L} \mathrm{ha}^{-1}$ ) com pulverizador costal motorizado marca Yamaho, modelo LS-937, com esguicho de bico duplo D-5 e pressão de $80 \mathrm{lbs} \mathrm{pol}^{-2}$. Foram avaliadas a produtividade total, comercial, extra AA e extra
A. Foram considerados frutos extra $A A$ aqueles com massa média maior que $150 \mathrm{~g}$ e frutos extra A os com massa média entre 100 e $150 \mathrm{~g}$.

A severidade da pinta preta foi avaliada semanalmente (BOFF, 1988) e integralizada como área abaixo da curva de progresso da doença (AACPD) de acordo com a fórmula $A A C P D=\Sigma$ $\left\{\left[\left(\mathrm{y}_{1}+\mathrm{y}_{2}\right) / 2\right]^{*} \Delta \mathrm{t}\right\}$, na qual y1 e y2 são duas avaliações sucessivas e $\Delta t$, o intervalo de tempo entre estas avaliações. As estimativas da taxa de progresso da doença ( $r$ ) foram obtidas com os valores de severidade ajustando-se ao modelo de Gompertz (CAMPBELL \& MADDEN, 1990).

Ensaio 2010. Os tratamentos avaliados foram: 1) pulverização semanal; 2) pulverização no acumulado de 10-VSD; 3) pulverização no acumulado de 15VSD; 4) pulverização no acumulado de 20-VSD; 5) pulverização no acumulado de 25-VSD; 6) pulverização no acumulado de 30-VSD; 7) testemunha sem pulverizar (inclusa no delineamento experimental) e quatro repetições. Cada parcela foi constituída de 12 plantas, sendo 10 úteis, espaçadas de $2,0 \mathrm{~m}$ entre fileiras e $0,54 \mathrm{~m}$ entre plantas. As mudas de tomate foram plantadas em 04/11/2010. Buscou-se avaliar a eficiência dos modelos de previsão com uso de um fungicida protetor, o clorotalonil (50\% i.a.), na dose de $150 \mathrm{~g}$ i.a.hl ${ }^{-1}$ em alto volume.

As avaliações de monitoramento das variáveis climáticas, aplicação das pulverizações, produção e intensidade da doença foram aquelas descritas na safra 2009-2010.

Ensaio 2011. Os tratamentos, avaliações de produção, severidade da doença, monitoramento das variáveis cli- 
máticas, a aplicação das pulverizações e determinação dos valores de severidade (VSD) foram aquelas descritas na safra 2010. As mudas de tomate foram plantadas em 25/10/2011. O fungicida boscalida (50\% i.a.) foi aplicado em cada tratamento semanal ou quando indicado pelo valor do respectivo VSD, na dosagem de 7,5g i.a. $\mathrm{hl}^{-1} \mathrm{em}$ alto volume.

\section{Resultadlos e discussão}

\section{Ensaio 2009}

Os primeiros sintomas da doença foram visíveis aos 56 dias após o plantio (DAP). A primeira pulverização no tratamento semanal ocorreu aos sete dias após o plantio (DAP). Nos tratamentos 10-VSD, 15-VSD, 20-VSD e 25-VSD a primeira pulverização ocorreu aos 33 DAP quando somatório do valor diário da severidade da doença totalizou o valor 35. O maior número de pulverizações ocorreu com o tratamento semanal totalizando 16 pulverizações, enquanto nos demais tratamentos houve redução em até $62,5 \%$ à medida que aumentava o nível do VSD. O intervalo médio entre uma pulverização e a seguinte foi de sete dias (semanal), nove dias (10-VSD 10), 11 dias (15-VSD), 14 dias (20-VSD) e 19 dias (25-VSD) e não houve diferença significativa $(P=0,283)$ na taxa de progresso da doença entre os tratamentos (Tabela 2) (Figura 2A).

A partir dos 70 DAP verificou-se uma maior frequência de valores de severidade 3 e 4 totalizando ao final do ciclo 202 VSDs acumulados (Figura 1A) e na severidade final não houve diferença significativa $(P=0,381)$ entre tratamentos. A área abaixo da curva do progresso da doença (AACPD) foi ligeiramente maior nos tratamentos 20 e 25-VSD, mas não houve diferença significativa $(P=0,193)$ entre estes e os demais tratamentos (Tabela 2) (Figura 2A).

Não houve diferença estatística significativa entre os tratamentos na produção total $(P=0,285)$, produção comercial, descarte e classificação comercial (Tabela 3). Em relação ao número de frutos nas classes comerciais extra $A A$ e extra $A$ também não houve diferença estatística significativa $(P=0,54 ; P=0,31$, respectivamente) entre os tratamentos (Tabela 3).

Tabela 2. Número de pulverizações (NP), severidade (SEV), área abaixo da curva (AACPD) e taxa do progresso $(R)$ da doença em função do modelo de previsão da pinta preta, em tomateiro cv. Paronset nos ciclos 2009, 2010 e 2011. Epagri, Caçador

Table 2. Number of sprays (NP), severity (SEV), area under disease progress curve (AACPD) and rate of progression $(R)$ of the disease as a function of the prediction model for the early blight in tomato cv. Paronset in the cycles 2009, 2010 and 2011. Epagri, Caçador

\begin{tabular}{|c|c|c|c|c|}
\hline Tratamentos & NP & SEV & AACPD & $\mathbf{R}$ \\
\hline & \multicolumn{4}{|c|}{2009} \\
\hline 10 vsd & 13 & 1,89 & 7,81 & 0,014 \\
\hline 15 vsd & 10 & 1,90 & 6,55 & 0,016 \\
\hline 20 vsd & 8 & 2,11 & 9,15 & 0,015 \\
\hline 25 vsd & 6 & 1,96 & 9,65 & 0,022 \\
\hline Semanal & 16 & 1,71 & 7,64 & 0,028 \\
\hline Teste $\mathrm{F}$ & - & $0,38^{\mathrm{ns}}$ & 0,19 ns & 0,28 ns \\
\hline \multirow[t]{2}{*}{ CV (\%) } & - & 14,15 & 23,05 & 50,77 \\
\hline & \multicolumn{4}{|c|}{2010} \\
\hline 10 vsd & 16 & 10,56 & 5,03 & 0,014 \\
\hline 15 vsd & 12 & 10,34 & 5,66 & 0,014 \\
\hline 20 vsd & 9 & 11,34 & 5,74 & 0,011 \\
\hline 25 vsd & 8 & 9,63 & 4,67 & 0,011 \\
\hline 30 vsd & 7 & 11,33 & 5,98 & 0,012 \\
\hline Semanal & 31 & 8,35 & 4,32 & 0,011 \\
\hline Testemunha & 0 & 13,13 & 6,35 & 0,013 \\
\hline Teste $\mathrm{F}$ & - & $0,35^{\text {ns }}$ & $0,88^{\mathrm{ns}}$ & $0,91^{\text {ns }}$ \\
\hline \multirow[t]{2}{*}{ CV (\%) } & - & 25,93 & 29,12 & 43,8 \\
\hline & \multicolumn{4}{|c|}{2011} \\
\hline $10 \mathrm{vsd}$ & 8 & 6,80 & $2,17 A^{1}$ & $0,028 A^{1}$ \\
\hline 15 vsd & 7 & 13,20 & $3,53 \mathrm{~B}$ & 0,034 B \\
\hline 20 vsd & 5 & 17,00 & $3,98 \mathrm{BC}$ & $0,038 \mathrm{C}$ \\
\hline 25 vsd & 5 & 16,20 & $3,72 \mathrm{~B}$ & $0,037 \mathrm{BC}$ \\
\hline $30 \mathrm{vsd}$ & 4 & 18,00 & $4,98 \mathrm{C}$ & $0,037 \mathrm{BC}$ \\
\hline Semanal & 25 & 5,40 & $1,35 \mathrm{~A}$ & $0,026 \mathrm{~A}$ \\
\hline Testemunha & 0 & 25,60 & $8,71 \mathrm{D}$ & $0,040 \mathrm{C}$ \\
\hline Teste F & - & $n s^{2}$ & $0,00001^{3}$ & $0,00001^{3}$ \\
\hline CV (\%) & - & - & 20,07 & 7,05 \\
\hline
\end{tabular}

${ }^{1}$ Médias seguidas da mesma letra na coluna não diferem pelo teste de Fischer a $5 \%$ de probabilidade; ${ }^{2}$ Teste não paramétrico de Kruskal-Wallis; ${ }^{3}$ Probabilidade do teste $\mathrm{F}$ altamente significativa

\section{Ensaio 2010}

Os primeiros sintomas da doença foram visíveis aos 85 DAP. A primeira pulverização do tratamento com calendário fixo (semanal) ocorreu aos 4 DAP. Para os sistemas de previsão (10-VSD, 15-VSD, 20-VSD, 25-VSD e 30-VSD) a primeira pulverização ocorreu aos 47 DAP.

O tratamento semanal totalizou 31 pulverizações. Já nos tratamentos com previsor, houve redução das pulverizações em relação à aplicação semanal de 48,38\% no 10-VSD (16 pulverizações), de $61,29 \%$ no 15 -VSD (12 pulveriza- ções); de 70,96\% no 20 -VSD (9 pulverizações); de $74,19 \%$ no 25 -VSD (8 pulverizações) e de $77,41 \%$ no 30 -VSD (7 pulverizações) (Tabela 2). O intervalo médio entre pulverizações foi de cinco dias com o calendário fixo (semanal), de oito dias no previsor 10-VSD, 12 dias no $15-V S D, 15$ dias no 20-VSD, 17 dias no 25 -VSD e 20 dias no $30-V S D$. O valor acumulado de VSD foi de 197 unidades para um período de cultivo de 140 dias (Figura 1B).

Houve uma tendência da testemunha absoluta em manter a maior seve- 


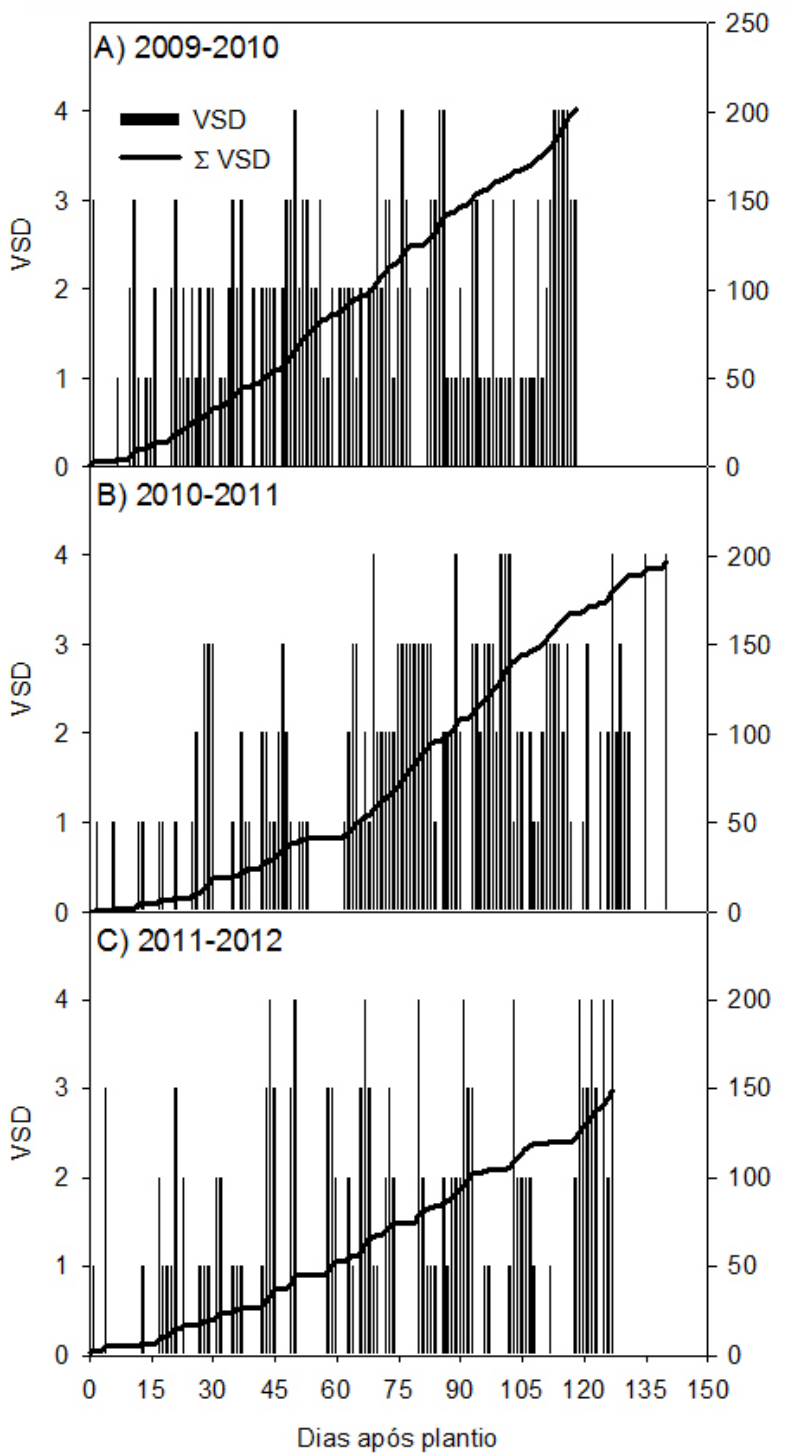

Figura 1. Valor de severidade diário (VSD) e acumulado ( $(\mathrm{VSD})$ da pinta-preta do tomateiro cv. Paronset durante os ciclos de cultivo 2009, 2010 e 2011. Epagri, Caçador

Figure 1. Daily severity value (VSD) and cumulative value (IVSD) of the early blight of tomato cV. Paronset during the 2009, 2010 and 2011 crop cycles. Epagri, Caçador

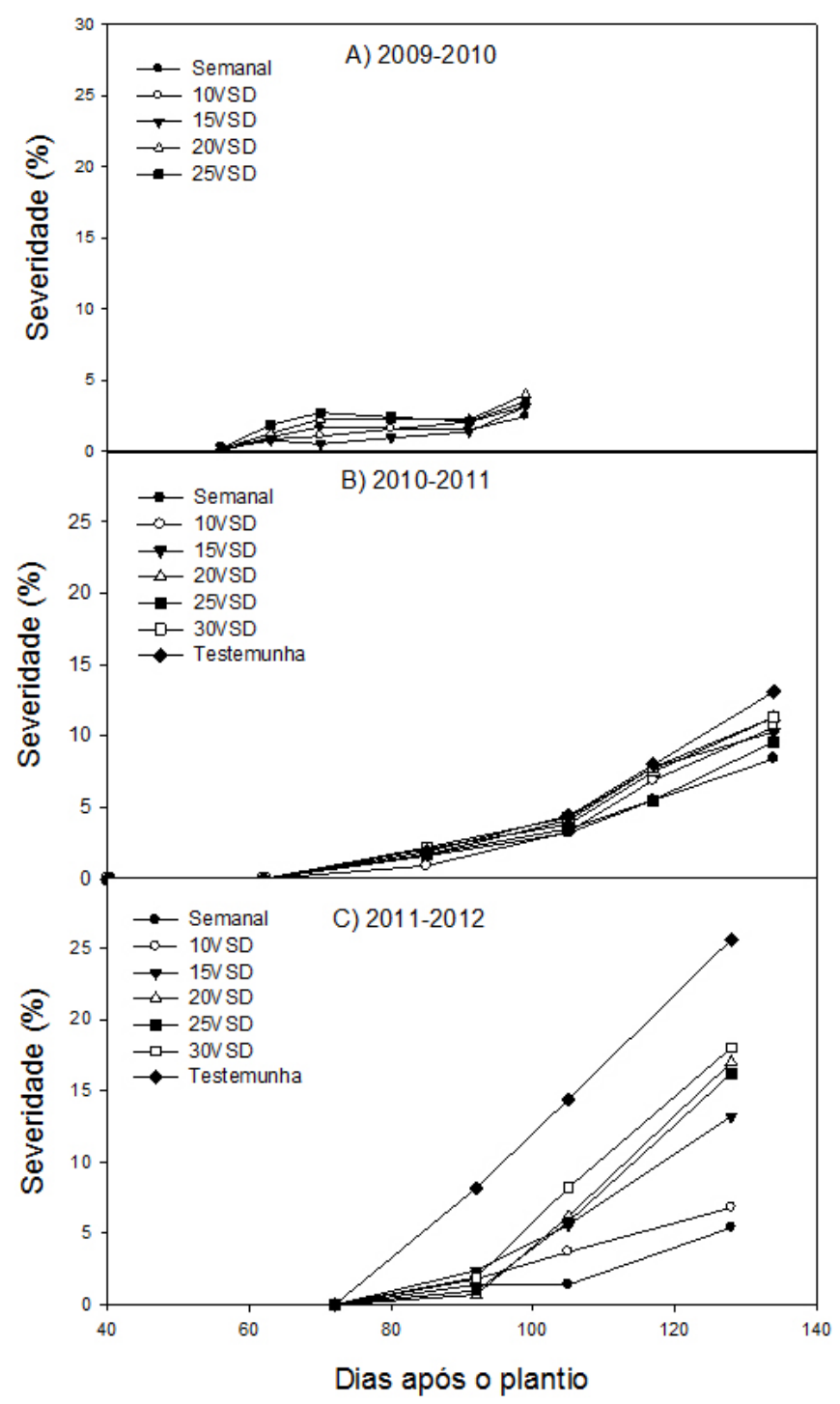

Figura 2. Curva de progresso da pinta preta em função do tratamento semanal e modelos de previsão (VSD) nos ciclos 2009, 2010 e 2011. Epagri, Caçador

Figure 2. Early blight progress curve as a function of weekly treatment and prediction models (VSD) in cycles 2009, 2010 and 2011. Epagri, Caçador ridade enquanto o tratamento semanal, a menor severidade (Figura 2B), contudo, esta diferença não foi significativa $(P=0,35)$ na avaliação final aos 134 DAP. Para as variáveis AACPD e taxa de progresso da doença também não foi observada diferença estatística significativa $(P=0,88$ e $P=0,91$, respectivamente) entre os tratamentos (Tabela 2).

Na produção total, produção comercial, descarte e classificação comercial não houve diferença estatística significativa $(P>0,05)$ entre os tratamentos (Tabela 3).

\section{Ensaio 2011}

Os primeiros sintomas da doença foram visíveis aos 92 DAP. A primeira pulverização do tratamento semanal ocorreu aos três DAP e totalizou 25 pulverizações. Nos tratamentos com previsor, a primeira pulverização ocorreu aos 44 DAP, totalizando no tratamento 10-VSD, oito pulverizações e redução de $68 \%$; no $15-V S D$, sete pulverizações e redução de $72 \%$; ambos os tratamentos 20-VSD e 25-VSD tiveram cinco pulverizações com redução de $80 \%$ e com 30 VSD houve apenas quatro pulverizações e redução de $84 \%$ (Tabela 2).

$\mathrm{O}$ intervalo médio entre pulverizações foi de cinco dias com o tratamento semanal, de 10 dias no previsor 10-VSD; de 12 dias no 15-VSD; de 17 dias nos tratamentos 20-VSD e 25-VSD e intervalo de 21 dias no 30-VSD. Esta maior amplitude de intervalo pode ter influenciado a taxa de progresso da doença, havendo diferença estatística $(P=0,00001)$ entre tratamentos; os tratamentos, semanal e 10-VSD, apresentaram uma menor taxa, diferindo dos demais, porém não entre si; já os demais, exceto o tratamento 15- 
Tabela 3. Produtividade de frutos total, comercial e descarte em função do modelo de previsão da pinta preta em tomateiro, cv. Paronset nos ciclos 2009, 2010 e 2011. Epagri, Caçador

Table 3. Total, commercial and discarded fruit productivity as a function of the prediction model of the early blight in tomato, cv. Paronset in the cycles 2009, 2010 and 2011. Epagri, Caçador

\begin{tabular}{|c|c|c|c|c|c|}
\hline Tratamentos & Total & Comercial & Extra AA & Extra A & Descarte \\
\hline & \multicolumn{5}{|c|}{$\mathrm{Mg} \mathrm{ha}^{-1}$} \\
\hline & \multicolumn{5}{|c|}{2009} \\
\hline 10 vsd & 79,27 & 76,35 & 38,15 & 38,20 & 2,92 \\
\hline 15 vsd & 70,67 & 67,88 & 38,45 & 29,43 & 2,79 \\
\hline 20 vsd & 73,34 & 69,65 & 37,33 & 32,32 & 3,69 \\
\hline 25 vsd & 68,74 & 65,07 & 34,30 & 30,77 & 3,67 \\
\hline Semanal & 73,33 & 69,40 & 36,46 & 32,94 & 3,93 \\
\hline Teste F & $0,28^{\text {ns1 }}$ & $0,19^{\text {ns }}$ & $0,54^{\mathrm{ns}}$ & 0,31 ns & $0,79^{\text {ns }}$ \\
\hline \multirow[t]{2}{*}{ CV (\%) } & 9,23 & 9,08 & 12,20 & 17,91 & 33,39 \\
\hline & \multicolumn{5}{|c|}{2010} \\
\hline 10 vsd & 76,94 & 67,77 & 53,80 & 13,97 & 9,17 \\
\hline 15 vsd & 79,57 & 71,19 & 57,01 & 14,18 & 8,38 \\
\hline 20 vsd & 72,61 & 62,17 & 47,61 & 14,56 & 10,44 \\
\hline 25 vsd & 77,04 & 67,26 & 56,10 & 11,16 & 9,78 \\
\hline 30 vsd & 77,51 & 68,18 & 55,22 & 12,96 & 9,33 \\
\hline Semanal & 75,11 & 67,87 & 53,72 & 14,15 & 7,24 \\
\hline Testemunha & 77,52 & 66,07 & 53,31 & 12,76 & 11,45 \\
\hline Teste F & $0,19^{\text {ns }}$ & $0,30^{\text {ns }}$ & $0,45^{\mathrm{ns}}$ & $0,98^{\text {ns }}$ & $0,063^{\text {ns }}$ \\
\hline \multirow[t]{2}{*}{ CV (\%) } & 13,40 & 14,63 & 16,90 & 17,83 & 18,77 \\
\hline & \multicolumn{5}{|c|}{2011} \\
\hline 10 vsd & 107,35 & 100,01 & 79,34 & 20,67 & 7,34 \\
\hline 15 vsd & 102,01 & 94,88 & 73,73 & 21,15 & 7,13 \\
\hline 20 vsd & 116,54 & 109,17 & 88,02 & 21,15 & 7,37 \\
\hline 25 vsd & 117,15 & 110,34 & 92,23 & 18,11 & 6,81 \\
\hline 30 vsd & 110,64 & 102,78 & 83,16 & 19,62 & 7,86 \\
\hline Semanal & 104,02 & 97,95 & 82,22 & 15,73 & 6,07 \\
\hline Testemunha & 101,47 & 94,42 & 77,19 & 17,23 & 7,05 \\
\hline Teste F & $0,29 \mathrm{~ns}$ & $0,31^{\mathrm{ns}}$ & $0,22^{\mathrm{ns}}$ & $0,06^{\mathrm{ns}}$ & $0,58^{\text {ns }}$ \\
\hline CV (\%) & 10,00 & 10,63 & 12,53 & 13,72 & 20,78 \\
\hline
\end{tabular}

1ns= teste $\mathrm{F}$ não significativo com probabilidade $\mathrm{P}>0,05$

VSD, a taxa de progresso da doença não diferiu da testemunha (Tabela 2). Devido à ocorrência de vários dias ao longo do ciclo, com a ausência de condição para a doença, o valor acumulado de VSD foi de 149 unidades, sendo o menor dos três ciclos avaliados (Figura 1C).

Em relação à severidade final, não se observou diferença entre os tratamentos, pela análise não paramétrica de Kruskal-Wallis. Entretanto, quando esta foi avaliada como a área abaixo da curva do progresso da doença (AACPD), houve diferença estatística significativa. A maior AACPD foi encontrada na testemunha e a diferença foi altamen- te significativa $(P=0,00001)$ dos demais tratamentos; a menor AACPD foi encontrada na pulverização semanal e 10-VSD diferindo dos demais tratamentos; os tratamentos 15-VSD, 20-VSD e 25-VSD, não diferiram entre si; o tratamento 20VSD também não diferiu do tratamento 30-VSD (Tabela 2).

Não houve diferença estatística significativa entre os tratamentos para as variáveis de produção total significativa $(P=0,29)$, produção comercial $(P=0,31)$, descarte $(P=0,58)$ e classificação comercial extra AA $(P=0,22)$ e extra $\mathrm{A}(P=0,06)$, (Tabela 3).

$\mathrm{O}$ período de cultivo do tomate em Caçador (SC) se estende de outubro a abril com condição ambiente propícia às principais doenças do tomateiro (WAMSER et al., 2008). O aumento de suscetibilidade à pinta preta está associado aos tecidos maduros: as folhas velhas são infectadas primeiramente e posteriormente as novas, após atingir maturidade fisiológica (JONES, 1991), sendo mais severa a partir da frutificação. Esta peculiaridade da doença permite que o acumulado de 35 valores de severidade como "gatilho" do primeiro previsor da doença do modelo TomCast seja admitido, também para a região de Caçador, uma vez que de 2009 a 2011 os primeiros sintomas ocorreram aos 56, 85 e 92 dias após o plantio, coincidindo com as primeiras colheitas e início da senescência do tomateiro.

As pulverizações subsequentes no modelo TomCast são indicadas com base no VSD igual a 20 (PITBLADO, 1992). Entretanto, na condição climática de Caçador, sugere-se que as pulverizações subsequentes à primeira ("gatilho") possam ocorrer quando o somatório atingir $25 \mathrm{VSD}$. O VSD igual a 25 proporcionou efetividade no controle da doença e significativa redução das aplicações desnecessárias durante o ciclo de cultivo com consequente redução de custo. Entretanto, estes valores de severidade devem ser validados na região na qual serão utilizados (CAMPBELL \& MADDEN, 1990).

A severidade foi baixa, não havendo evidência de efeito sobre a produção mesmo quando a severidade na testemunha atingiu 25,5\%. Basu (1974) demonstrou que sem a aplicação de fungicidas, $60 \%$ da área foliar infectada 
seriam necessários para causar lesões em $10 \%$ dos frutos e o comprometimento de 10 a $30 \%$ no número de frutos comerciais. Segundo Vloutoglou \& Kalogerakis (2000) e Chelal et al. (2015), à medida que aumenta a maturidade das plantas, a porcentagem de desfolha pela doença também aumenta. Apenas no ensaio 2012 houve diferença na AACPD e taxa de progresso da doença, enquanto as demais variáveis avaliadas não diferiram entre os tratamentos. Considerando que o objetivo do trabaIho foi utilizar um modelo de previsão para o controle da doença e redução do número de aplicações, isso foi alcançado, em maior proporção, com os tratamentos 20-VSD, 25-VSD e 30-VSD em semelhança aos dados encontrados por Paul et al., (2004).

Modelos de previsão de doenças no Brasil ainda são pouco utilizados, não devido ao custo dos equipamentos agrometeorológicos, mas à falta de pesquisas de campo. Em Santa Catarina, após a validação deste modelo de previsão, na unidade piloto da produção integrada (Sispit), o Ciram/Epagri disponibilizou-o na plataforma Agroconnect sem nenhum custo ao tomaticultor, auxiliando-o na decisão do momento de aplicação do fungicida no controle da pinta preta (Alternaria spp.)

\section{Conclusão}

O uso de sistema de previsão TomCast permitiu reduzir significativamente o número de aplicações de fungicidas, sem afetar a produtividade ou qualidade dos frutos. Estes valores de severidade (VSD) poderão ser validados em acordo com as exigências locais do cultivo.

\section{Referências}

BASU, P.K. Measuring early blight, its progress and influence on fruit losses in nine tomato cultivars. Canadian Plant Disease Survey, v.54, n.2, p.45-51, 1974.

BOFF, P. Epidemiologia e controle químico da mancha-de-estenfílio (Sthemphylium solani Weber) e da pinta preta (Alternaria solani Jones \& Grout), em dois sistemas de condução do tomateiro (Lycopersicon escu- lentum Mill). 140p.1988. Dissertação (Mestrado) - Universidade Federal de Viçosa, Viçosa, MG,1988.

CAMPBELL, C.L.; MADDEN, L.V. Introduction to plant disease epidemiology. New York: John Wiley \& Sons, 1990. 532p.

CHELAL, J.; MASRI, A.A.; HAU, B. Modelling the interaction between early blight epidemics and host dynamics of tomato. Tropical Plant Pathology, Brasília, v.40, n.2, p.77-87, 2015.

DORMAN, E. A.; WEBSTER, B. J.; HAUSBECK, $M$. K. Managing foliar blights on carrot using copper, azoxystrobin, and chlorothalonil applied according to TOM-CAST. Plant Disease, St. Paul, v.93, n.4, p.402-407, 2009.

FAORO, I. D.; TOMASELLI, A. (Coords.). Normas técnicas para o tomateiro tutorado na região do Alto Vale do Rio do Peixe. Florianópolis: Epagri. 60p. (EPAGRI. Sistemas de Produção, 27).

JONES, J.P. Early Blight. In: JONES, J.B; JONES, J.P.; STALL, R.E.; ZITTER, T.A. (Eds.) Compedium of tomato diseases. St. Paul: The American Phytopathological Society, p.13-14, 1991.

LOPES, C.A.; ÁVILA, A.C. Doenças do tomateiro. Brasília: Embrapa Hortaliças, 2005 151p.

IBGE. Levantamento Sistemático da Produção Agrícola: pesquisa mensal de previsão e acompanhamento das safras agrícolas no ano civil. Rio de Janeiro: IBGE, v.30, n.1, p.181, jan. 2017.

MADDEN, L.V.; PENNYPACKER, S.P.; MACNAB, A.A. Fast - a forecast system for Alternaria solani on tomato, Phytopathology, St. Paul, v.68, n.9, p.1354-1358, 1978.

MUELLER, S.; WAMSER, A.F.; BECKER, W.F.; SANTOS, J.P. Indicações técnicas para o tomateiro tutorado na Região do Alto Vale do Rio do Peixe. Florianópolis: Epagri, 2008. 78p. (Epagri. Sistemas de Produção, 45).

PANDOLFO, C.; BRAGA, H.J.; SILVA JÚNIOR, V.P., MASSIGNAN, A.M.; PEREIRA, E.S.; THOMÉ, V.M.R; VALCI, F.V Atlas climatológico do Estado de Santa Catarina. Florianópolis: Epagri. [CD-ROM], 2002.

PAUL, P.A.; VALE, F.X.R.; ZAMBOLIM, L. FONTES, P.C.R.; COELHO, R.R.; MACABEU, A.J.
Epidemiologia comparativa da pinta preta do tomateiro sob quatro regimes de pulverização. Fitopatologia Brasileira, Brasília, v.29, n.5, p. 475-479, 2004.

PITBLADO, R.E. The development and implementation of TOMCAST- a weathertimed fungicide spray program for field tomatoes. Ridgetown: Ministry of Agriculture and Food; Ridgetown College of Agricultural Technology, 1992. 22p.

RODRIGUES, T.T.M.S. Morphological, molecular characterization, and inference about recombination, for species of Alternaria related to early blight of potato and tomato. 78p. 2009. Tese (Doutorado) - Universidade Federal de Viçosa, Viçosa, MG, 2009.

SANTOS, J. R. M. dos. Desenvolvimento do sistema de previsão e aviso "PAST" para requeima (Phytophthora infestans (Mont.) de Bary), pinta preta (Alternaria solani (Ellis \& Martin) L. R. Jones) e septoriose (Septoria lycopersici Speg.) em tomateiro e sua avaliação no controle da pinta preta. 129p. 2000. Tese (Doutorado) - Universidade de Brasília, Brasília, DF, 2000.

SIKORA, E.J.; KEMBLE, J.M.; ZEHNDER, G.W.; GOODMAN, W.R.; ANDRIANIFAHANANAET, M.; BAUSKE, E.M. Using on-farm demonstrations to promote integrated pest management practices in tomato production. HortTechnology, Alexandria, v.12, n.3, p.485488, 2002.

TÖFOLI, J.G.; DOMINGUES, R.J.; GARCIA JUNIOR, O.; KUROZAWA, C. Controle da pinta preta do tomateiro por fungicidas e seus impactos na produção. Summa Phytopathologica, Botucatu, v.29, n.3, p.225-233, 2003.

VLOUTOGLOU, I.; KALOGERAKIS, S. N. Effects of inoculum concentration, wetness duration and plant age on development of early blight (Alternaria solani) and on shedding of leaves in tomato plants. Plant Pathology, London, v.49, n.1, p.339-345, 2000.

WAMSER, A.F; BECKER, W.F.; SANTOS, J.P.; MUELLER, S. Influência do sistema de condução do tomateiro sobre a incidência de doenças e insetos-praga. Horticultura Brasileira, Brasília, v.26, n.2, p.180-185, 2008

ZADOKS, J.C.; SCHEIN, R.D. Epidemiology and Plant Disease Management. New York: Oxford University Press. 1979. p.119-170. 